\title{
Transiently Trapped Entanglements in Model Polymer Networks
}

\author{
Rodolfo H. Acosta, ${ }^{\dagger}$ Gustavo A. Monti, ${ }^{\dagger}$ Marcelo A. Villar, ${ }^{\S}$ Enrique M. Vallés, ${ }^{\S}$ and \\ Daniel A. Vega* $*$ \\ ${ }^{\dagger}$ Facultad de Matemática, Astronomía y Física (UNC), Instituto de Física, IFFAMAF (UNC-CONICET), \\ Medina Allende s/n, Ciudad Universitaria, 5016 Córdoba, Argentina ${ }^{\S}$ Planta Piloto de Ingeniería Química, \\ PLAPIQUI (UNS-CONICET), Camino La Carrindanga Km 7, CC717, 8000 Bahía Blanca, Argentina, and \\ "Department of Physics, Universidad Nacional del Sur, CONICET, Av. L. N. Alem 1253, 8000 Bahía Blanca, \\ Argentina
}

Received November 13, 2008; Revised Manuscript Received May 13, 2009

\begin{abstract}
The relaxational dynamics of trapped entanglements in model silicone polymer networks is studied through the residual dipolar couplings (RDC) obtained by double quantum nuclear magnetic resonance (DQ NMR). These experiments were performed on model polymer networks containing linear pendant chains. The model networks where synthesized by end-linking a mixture of $\alpha$, $\omega$-divinyl poly (dimethylsiloxane) $\left(\mathrm{B}_{2}\right)$ and $\omega$-vinyl poly(dimethylsiloxane) $\left(\mathrm{B}_{1}\right)$ with trifunctional $\left(\mathrm{A}_{3}\right)$ or tetra-functional $\left(\mathrm{A}_{4}\right)$ cross-linkers. At the time scale of the NMR experiments only a small fraction of the linear pendant chains $\mathrm{B}_{1}$ loses the memory of its early configuration. Then, the unrelaxed topological constrains involving pendant material render a nonzero average dipolar coupling that contributes to the solid-like behavior of the NMR response. Irrespective of the functionality of the cross-linkers, upon the presence in the network of pendant chains induced by the insertion of the $\mathrm{B}_{1}$ monofunctional poly(dimethylsiloxane) an important reduction in the RDC is observed as a consequence of the transiently trapped entanglements. It was also verified that, according to the viscoelastic response, the networks prepared with $\mathrm{A}_{4}$ cross-linkers show systematically higher values of the residual dipolar couplings than trifunctional cross-linked networks.
\end{abstract}

\section{Introduction}

Over the last 5 decades the dynamic response of entangled polymer melts has been an outstanding problem in polymer science. Most of the viscoelastic and diffusive properties of polymer melts and concentrated polymer solutions are profoundly influenced by topological interactions ${ }^{1}$. The most successful model to deal with topological constrains is the tube model. ${ }^{2}$ According to this model, the topological confinement exerted on a given molecule by the surrounding media can be modeled as a hypothetical tube that severely suppress the motion perpendicular to the tube's local axis, but permits the diffusion along the tube.

In the case of entangled linear polymer melts, the dominant mechanism of relaxation at long times is the reptation of the molecule in the confining tube. Although this mechanism may be also important in the diffusional motion of complex branched molecules, like $\mathrm{H}$ or comb shaped polymers, in this case two additional mechanisms must be considered in order to account for the experimental observations: contour-length fluctuations of the polymer chain in its own confining tube and constraint-release. ${ }^{1,2}$ Contour length fluctuations account for the unconstrained diffusive transport of polymer segments along the primitive path (the primitive path means the shortest path connecting the two ends of the chain respecting the topological constrains upon it). The constrain release mechanism takes into account the fact that some of the chains that define the confining tube release their constraints by bringing one of the free ends into the tube.

One of the most successful approaches to deal with the constrain release problem is the tube dilution concept, originally introduced for entangled polymer melts by Marrucci in $1985^{3}$ According to this approach, fast relaxing segments in a polymer

*Corresponding author. E-mail address: dvega@criba.edu.ar. melt act as solvent for the slower relaxing chain segments and the tube progressively widens as entanglements are released by other relaxing neighboring polymer chains. As a consequence of the dynamic dilution effect, as time proceeds the effective primitive path length becomes smaller and the relaxational dynamics becomes faster. ${ }^{4,5}$

Irrespective of the fact that an appreciable disagreement between dielectric spectroscopy measurements and theoretical predictions has been observed by some authors, ${ }^{1,5}$ the tube dilution concept has proved to work remarkably well to describe the viscoelastic response of different entangled branched structures. ${ }^{5-11}$

Considering that constrain release or dynamic dilution concepts rely on the hypothetical tube representing the invariant topological constrains of the surrounding chains, the study of the chain dynamics in polymer networks is very important. ${ }^{12,13}$ Since in polymer networks the process of dynamic dilution is inhibited (the tube diameter remains roughly constant during the chain diffusion process), well characterized polymer networks containing guest chains of a given architecture become an ideal model system to test the validity of different theories. ${ }^{14,15}$ For example, by preparing poly(dimethylsiloxane) networks with different mesh sizes, Urayama et al. have studied the dynamics of guest linear polymers. ${ }^{12}$ These authors do not only find that the dynamics of the guest chains can be well described by the original tube model, but also that the response is strongly retarded when the mesh size, $M_{x}$, is below the average molecular weight between entanglements, $M_{e}$. The consequences of the invariant topology of polymer networks onto the dynamics of branched structures has also been analyzed by studying the viscoelasticity of dangling chains and guest symmetric star polymers. ${ }^{13}$ In this work, by using well characterized silicone networks with controlled amounts of linear and star-shaped defects, not only the absence of dynamic dilution effects but also the equivalence between the 
relaxational dynamics of stars and linear dangling chains trapped into the network have been recently verified. Differently from linear entangled polymers, reptation is inhibited for branched polymers and stress mainly relaxes by arm retraction ${ }^{16}$. By this mechanism, the free chain end fluctuates down its tube some distance toward the branching point and pops out again, thus loosing the memory of its initial configuration in the section of tube close to the free end. This process is entropically unfavorable and the time scale for retraction increases roughly exponentially with the size of the branch. ${ }^{1,5,16}$

To prepare polymer networks containing guest molecules, the end-linking method is more advantageous than the conventional random cross-linking method because it allows not only to control the mesh size, but also to reduce the content of undesired soluble and pendant material. ${ }^{12,13}$ In this kind of reaction, the end functional groups of linear telechelic chains are reacted with cross-linking agents having a functionality $f$, where $f$ is an integer $(f>2)$. If every telechelic prepolymer chain has two terminal reactive groups $\left(\mathrm{B}_{2}\right)$, and each group effectively reacts with different cross-linker molecules $\left(\mathrm{A}_{f}\right)$, then an ideal or model network is obtained. In this case, the average chain size $M_{x}$ between cross-linking points is equal to the length of the bifunctional $\mathrm{B}_{2}$ molecules used to prepare the model network. Note that the control of the content of network defects generated by incomplete reaction is very important. Unreacted ends give origin to branched pendant chains and isolated molecules. Since both, pendant and branched solubles have a star-like relaxational behavior with long relaxation times, these undesired defects can easily hide the contribution associated to the guest chains.

Rheology and nuclear magnetic resonance (NMR) are excellent techniques for the study of elastomers. ${ }^{17}$ As the mechanical properties of polymer networks are dominated by motions that range from sequences of a number of monomers units up to the whole chain, NMR methods are suitable to probe relaxational processes at relatively small time scales. It was very early suggested by Cohen Addad $^{18}$ that the anisotropic chain motion in molten polymers followed a solidlike behavior, that is, that the topological restrictions rendered a nonzero average dipolar coupling that could influence the NMR signals in transverse relaxation experiments. The relaxation of ${ }^{1} \mathrm{H}$ transverse magnetization is mainly determined by the dipole-dipole magnetic interaction between protons. This interaction is modulated at different extents by molecular motions, and therefore, it is sensitive to differences in the motion of the chains that form the polymer network. This technique has been shown to give a very precise measure of the amount of entangled polymer chains that form the elastic network and the pendant material. At the normal NMR time scales, the elastic chains behave as solidlike while the pendants chains behave as isotropic ones. ${ }^{19}$ Nevertheless, the extraction of dipolar couplings is subject to strong model assumptions and additionally is coupled to other parameters. The use of multiple quantum NMR (MQ NMR) has proven to be more adequate to probe residual dipolar couplings; in particular double quantum NMR under static or fast rotating conditions is used to obtain dipolar couplings by inspection of the short time behavior of the DQ coherences. ${ }^{20-23}$

In this work we use static DQ NMR experiments to study the dynamic response of polymer networks containing defects of well characterized structure. For $\mathrm{A}_{f}+\mathrm{B}_{2}$ systems $(f=3,4)$, at complete reaction the only way to obtain pendant chains is to prepare formulations with an initial stoichiometric imbalance, $r$. In this case, the amount of dangling material that remains in the system at the end of the reaction depends on $r^{24-26}$. The principal disadvantage of these systems is that the resultant pendant chains have complex branched structure with a wide variety of relaxational mechanisms. Consequently, it is extremely difficult to correlate the experimental data with the structure of the network.

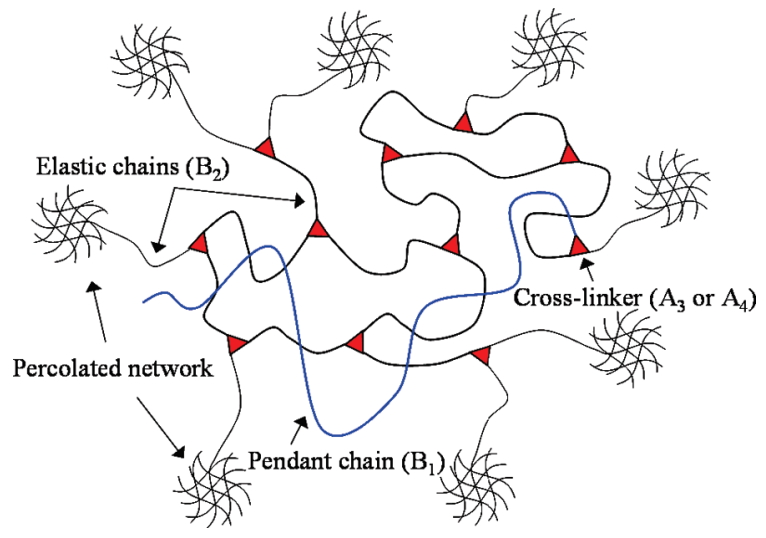

Figure 1. Schematic representation of the polymer networks employed in this study. Note that linear pendant chains $\left(B_{1}\right)$ are linked to the network structure only through one of the chain ends. In this study we employed tri- $\left(\mathrm{A}_{3}\right)$ or tetra-functional $\left(\mathrm{A}_{4}\right)$ cross-linkers.

For this reason, we have chosen to study the relaxation behavior of pendant chains employing model networks with controlled amounts of monodisperse linear chains dangling from the gel structure. ${ }^{13}$ These networks are prepared by reacting a mixture of a bifunctional prepolymer with reactive functional groups at the chain ends $\left(\mathrm{B}_{2}\right)$, with linear monodisperse chains of the same polymer with only one functional end group $\left(\mathrm{B}_{1}\right)$, and a polyfunctional cross-linker $\left(\mathrm{A}_{f}\right)$. The final structure of the network obtained by this procedure depends on the relative amounts in which these components are mixed in the initial formulation of the crosslinking reaction. The cartoon shown in Figure 1 presents a schematic representation of the model network employed in this study.

\section{Materials and Methods}

2.1. Molecular Structure. Model poly(dimethylsiloxane) networks were obtained by the hydrosylilation reaction, based on the addition of the hydrogen atom from the silane groups belonging to the cross-linker molecules to the end vinyl groups present in the telechelic prepolymer molecules ${ }^{24}$. A commercial difunctional prepolymer, $\alpha, \omega$-divinyl poly(dimethylsiloxane) $\left(\mathrm{B}_{2}\right)$ (United Chemical Technology, Inc.) and a quasi-monodisperse monofunctional prepolymer, $\omega$-vinyl poly(dimethylsiloxane) $\left(B_{1}\right)$ were used. Trifunctional phenyltris(dimethylsiloxy) silane $\left(\mathrm{A}_{3}\right)$ and tetrafunctional tetrakis(dimethylsiloxy)silane $\left(\mathrm{A}_{4}\right)$ were employed as cross-linkers. A Pt salt was utilized as a homogeneous catalyst for the hydrosilylation reaction. The $\mathrm{B}_{1}$ prepolymers were synthesized by anionic polymerization of hexamethylciclotrisiloxane as reported elsewhere ${ }^{25}$.

Networks prepared by adding small amounts of $\mathrm{B}_{1}$ monofunctional chains to the reacting mixture of $\mathrm{B}_{2}$ and the proper cross-linker in a stoichiometrically balanced system contain a controlled content of pendant chains ${ }^{13,24}$. A $20 \mathrm{wt} \%$ of $\mathrm{B}_{1}$ chains was added to all networks except for two tri- and tetrafunctional reference networks that do not contain $\mathrm{B}_{1}$ chains. An average molecular weight of $M_{\mathrm{wB} 2}=23900 \mathrm{~g} / \mathrm{mol}$ for the $\mathrm{B}_{2}$ chains was used. Other relevant parameters of the experimental characterization of the networks are listed in Table 1. Networks were subjected to extraction of solubles using toluene as solvent by the procedure described elsewhere ${ }^{25,26}$. After soluble extraction, the mass fraction of elastic $\left(W_{e}\right)$ and pendant chains $\left(W_{p}=1-W_{\mathrm{e}}\right.$ ) were determined through mean field calculations (see Appendix I), considering the final advance of reaction reached in each network (Table 1). These calculations indicate that pendant material is mainly constituted by reacted $B_{1}$ chains and partially reacted $\mathrm{B}_{2}$ chains. ${ }^{25}$

2.2. Double Quantum NMR. NMR measurements were performed with a Bruker Avance II spectrometer operating at a resonance frequency of $300.13 \mathrm{MHz}$ for protons at a temperature of $303 \mathrm{~K}$. A DOTY DSI-703 proton dedicated probe with proton 
Table 1. Network Functionality $(f)$, Monofunctional Chain Molecular Weight $\left(M_{\mathrm{wB} 1}\right)$, Weight Fraction of $\mathrm{B}_{1}$ Chains $\left(W_{\mathrm{B} 1}\right)$, Fraction of Solubles $\left(W_{s}\right)$, Extent of Reaction $(p)$ and the Fraction of Pendant chains $\left(W_{p}\right)$, As Determined by Mean Field Theory (See Appendix I $)^{a}$

\begin{tabular}{|c|c|c|c|c|c|c|c|c|c|}
\hline$f$ & $M_{\mathrm{wB} 1}(\mathrm{~g} / \mathrm{mol})$ & $W_{\mathrm{B} 1}$ & $W_{s}$ & $p$ & $W_{p}$ & $D_{\text {res }} / 2 \pi[\mathrm{Hz}]$ & $D_{G} / 2 \pi[\mathrm{Hz}]$ & $\sigma_{G} / 2 \pi[\mathrm{Hz}]$ & $\chi$ \\
\hline 3 & & & 0.007 & 0.937 & 0.145 & 189 & 189 & 13 & 0.147 \\
\hline 3 & 26500 & 0.202 & 0.049 & 0.906 & 0.401 & 143 & 145 & 22 & 0.206 \\
\hline 3 & 51300 & 0.201 & 0.043 & 0.906 & 0.386 & 154 & 155 & 20 & 0.395 \\
\hline 3 & 60600 & 0.199 & 0.041 & 0.880 & 0.369 & 161 & 164 & 25 & 0.427 \\
\hline 3 & 83500 & 0.201 & 0.039 & 0.892 & 0.364 & 159 & 162 & 28 & 0.478 \\
\hline 3 & 121300 & 0.199 & 0.039 & 0.896 & 0.367 & 159 & 161 & 25 & 0.523 \\
\hline 4 & & & 0.004 & 0.933 & 0.124 & 216 & 219 & 36 & 0.147 \\
\hline 4 & 26500 & 0.217 & 0.044 & 0.866 & 0.384 & 161 & 167 & 39 & 0.204 \\
\hline 4 & 51300 & 0.203 & 0.051 & 0.854 & 0.403 & 164 & 171 & 47 & 0.358 \\
\hline 4 & 60600 & 0.209 & 0.038 & 0.871 & 0.368 & 181 & 187 & 38 & 0.475 \\
\hline 4 & 83500 & 0.214 & 0.030 & 0.878 & 0.346 & 184 & 189 & 38 & 0.477 \\
\hline 4 & 121300 & 0.221 & 0.036 & 0.888 & 0.371 & 186 & 191 & 37 & 0.514 \\
\hline
\end{tabular}

${ }^{a}$ The last columns include the values obtained by fitting the NMR data with eqs 1 and 2 and the fraction of unrelaxed material $\chi$ calculated with eq $5 \mathrm{~B}$.

background signal reduction was used. Slices of $1 \mathrm{~mm}$ of sample were centered in $4 \mathrm{~mm}$ outer diameter sample holders in order to maximize the homogeneity of the radio frequency field.

A MQ selection scheme of excitation/reconversion periods of equal duration $\tau$ is done with a pulse sequence that excites all even quantum orders in a multispin system. ${ }^{27}$ The modification to the sequence proposed by Baum et al. ${ }^{28}$ and the normalization procedure introduced by Saalwachter ${ }^{22}$ is used. Excitation and reconversion blocks are generated by the repetition $n_{\mathrm{c}}$ times of an eight $90^{\circ}$ pulse scheme, together with the introduction of four $180^{\circ}$ pulses per block that compensate for better offset and pulse imperfection. The total unitary block duration is $t_{\mathrm{c}}=240 \mu \mathrm{s}$. Finite pulse effects are taken into consideration by scaling with a duty cycle dependent factor $\xi=1-12 t_{\mathrm{p}} / t_{\mathrm{c}}$, with $t_{\mathrm{p}}=2 \mu \mathrm{s}$ being the duration of the $90^{\circ}$ pulse. Therefore, MQ coherences are excited with a variable time $\tau=\xi n_{\mathrm{c}} t_{\mathrm{c}}$. Detection of the MQ encoded signal is performed by a single $90^{\circ}$ pulse applied after a z-filter time $t_{z}=10 \mathrm{~ms}$, which is approximately $5 T_{2}{ }^{*}$. The evolution time between excitation/reconversion blocks was set to $1 \mu \mathrm{s}$. Two sets of experiments are performed, on the first experiment the receiver phase is cycled in $\pm \pi$ on each acquisition, thus detecting $4 n+2 \mathrm{MQ}$ coherences.

For excitation times much shorter than the transverse relaxation time it has been shown that for soft elastomers only DQ coherences are detected, which on the following are referred to as $S_{D O}{ }^{22}$. The second experiment, without receiver cycling, contains contributions from all $4 n$ quantum orders as well as dipolar encoded longitudinal magnetization, plus signal from dangling segments which appear as isotropically mobile components, $S_{r e f}$. The quantity $S_{\Sigma}=S_{D Q}+S_{r e f}$ contains all the dipolar refocused magnetization of the sample. Finally $S_{n D Q}=S_{D Q} / S_{\Sigma}$, is used to remove the influence of relaxation from the data. These signals are plotted in Figure 2 for a network with no addition of $\mathrm{B}_{1}$ chains and $\mathrm{A}_{3}$ cross-linker. The scale corresponds to the intensity of a signal acquired after a single $90^{\circ}$ pulse, which contains contributions from both elastic and dangling chains. The influence of this last type of chains, which can be regarded as liquid-like components, is clearly depicted by the fact that $S_{n D Q}$ does no reach and level off at the theoretical 0.5 value $^{22}$. In order to remove the influence of liquid-like segments in the normalization procedure, we applied a DQ filter ${ }^{22,27,29}$ which consists of introducing an extra pair of excitation/reconversion blocks before the one used to record the buildup curves, of fixed duration $\tau_{\max }^{f}$ corresponding to the maxima of $S_{D Q}$. The time interval between the two excitation/reconversion block pairs was set to $t_{z}$. Thus, the second block-pair can be used to study the DQ buildup, $S_{D O}^{f}$ of the preselected dipolar encoded signals only. Two independent four step phase cycles are then applied to the first and second pair of DQ excitation/reconversion, which turns out in a 16-step phase cycle. Figure 2 also shows the results for $\tau_{\max }^{f}=3 \mathrm{~ms}\left(n_{\mathrm{c}}=14\right)$. The normalized filtered signal, $S_{n D Q}^{f}$, is seen to reach the expected plateau of 0.5 .

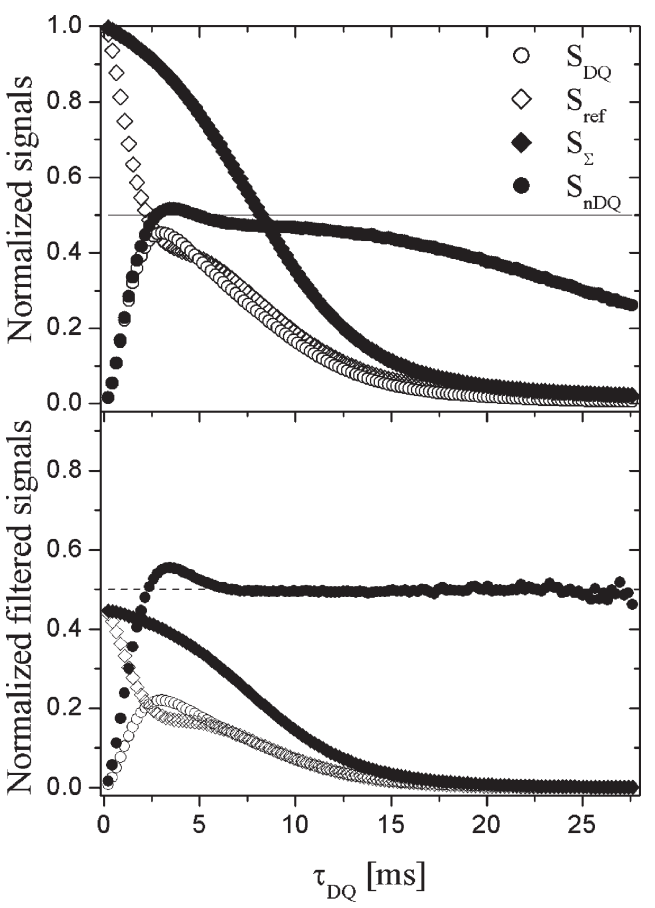

Figure 2. Experimental data from MQ experiments for a trifunctional network. Top: nonfiltered signals. Bottom: DQ prefilter normalized signals. Relaxation is present in nonfiltered data for times longer than 6 $\mathrm{ms}$ and it is completely removed in prefiltered data.

2.3. Residual Dipolar Couplings. Using intensities $S_{n D Q}^{f} \leq 0.45$ the data are fitted with an inverted Gaussian function based on static second moment approximations: ${ }^{22}$

$$
S_{n D Q}^{f}\left(D_{\text {res }}\right)=\frac{1}{2}\left[1-\exp \left(\frac{2}{5} D_{\text {res }}{ }^{2} \tau^{2}\right)\right]
$$

Here, $D_{\text {res }}$ is an apparent residual dipolar coupling that represents an average over different internuclear spin pair couplings. It has been shown that deviation from an inverted Gaussian indicates the presence of substantial distribution of dipolar couplings. If the distribution is assumed to be Gaussian an average RDC $\left(D_{G}\right)$ as well as the standard deviation $\left(\sigma_{G}\right)$ can be obtained by ${ }^{30,31}$

$$
S_{n D Q}^{f}\left(D_{G}, \sigma_{G}\right)=\frac{1}{2}\left[1-\frac{\exp \left(-\frac{\frac{2}{5} D_{G}^{2} \tau^{2}}{1+\frac{4}{5} \sigma_{G}{ }^{2} \tau^{2}}\right)}{\sqrt{1+\frac{4}{5} \sigma_{G}^{2} \tau^{2}}}\right]
$$

Furthermore, numerical inversion methods can be used to obtain the distribution of dipolar couplings. Nevertheless, as 
shown in the following section, the representation provided by eq 1 is accurate enough for the samples used in this work due to the high degree of control on the reactions during the synthesis of the different polymer networks. ${ }^{24}$

\section{Results and Discussion}

The average RDCs obtained by fitting the DQ filtered build up curves with eqs 1 and 2 are listed in Table 1 . It can be observed that the values of $D_{\text {res }}$ are in good agreement with those of $D_{G}$, indicating that a narrow distribution of couplings is present on the samples used in this work. The ratios $\sigma_{G} / D_{G}$ are lower than 0.3 for all samples, validating the accuracy of the Gaussian fitting; ${ }^{31}$ additionally, it supports the use of a prefilter. If a broad distribution is present, the action of a prefilter can select very specific components from the overall distribution. ${ }^{30}$ It is worth to mention (data not shown here) that the same values are obtained with 5\% dispersion for several significant networks measured with the non DQ filtering scheme and processed by the most popular tail subtraction method. ${ }^{22,30,31}$ This observation assures that the powder average is recovered to a very good extent by the chosen $t_{z}$-filter time. ${ }^{30}$

Figure 3 shows the obtained $D_{\text {res. }}$ Note that networks prepared with $\mathrm{A}_{4}$ cross-linker show systematically higher values of the $\mathrm{RDC}$, i.e., larger elasticity, in qualitative agreement with the predictions of the phantom network theory of rubber elasticity. In addition, as compared with $\mathrm{B}_{1}$ free networks, upon the addition of monofunctional pendant chains an important reduction in $D_{\text {res }}$ is observed. Two main mechanisms are responsible for this reduction in the elastic response: (a) In stoichiometrically balanced systems, the addition of each $B_{1}$ chain leads to the annihilation of one of the effective point of the cross-linker $\left(\mathrm{A}_{3}\right.$ networks) or to a reduction in the cross-linker effective functionality ( $\mathrm{A}_{4}$ networks). Then, the density of elastically active crosslinkers must decrease as $\sim 1 / M_{\mathrm{wB} 1}$. (b) The addition of a $20 \%$ of $\mathrm{B}_{1}$ chains reduce the concentration of elastically active chains (see also Appendix I), and then also $D_{\text {res }}$.

Although $\mathrm{B}_{1}$ chains modify the network structure by eliminating a certain number of elastically active cross-linkers, its effect as "dynamic diluent" depends on the time scale considered. ${ }^{13}$ At very long times, $B_{1}$ chains do not participate to the elastic response, and its net effect is to swell the network and to reduce its elastic modulus. However, at intermediate times the memory of the pendant material is not completely lost and transiently trapped entanglements also contribute to the elastic response. Since the relaxation time of the pendant material has a very strong dependence on its molecular weight, ${ }^{13}$ at the typical DQ NMR time scales the fraction of unrelaxed material also contributes to $D_{\text {res }}$. This behavior can be observed in Figure 3, where for fixed network functionality, $D_{\text {res }}$ increases as $M_{\mathrm{wB} 1}$ increases.

We can note that in order to describe the behavior of polymer networks containing pendant material through $D_{\text {res }}$, three main components should be taken into account:

(a) Elastically active chains (both chain ends connected to the network): since we are dealing with networks well beyond the critical gel point, the weight fraction of elastically active material, $W_{e}$, can be accurately determined through mean field theory. ${ }^{19,24}$ According to this model, the fraction of elastically active material can be obtained from the initial formulation of the reactants and the maximum extent of reaction obtained experimentally from the weight fraction of soluble material (for more details see Appendix I).

(b) Permanently trapped entanglements (four paths leading away from an entanglement site between two chains lead independently to the network): Although the presence of permanently trapped entanglements is completely unavoidable,

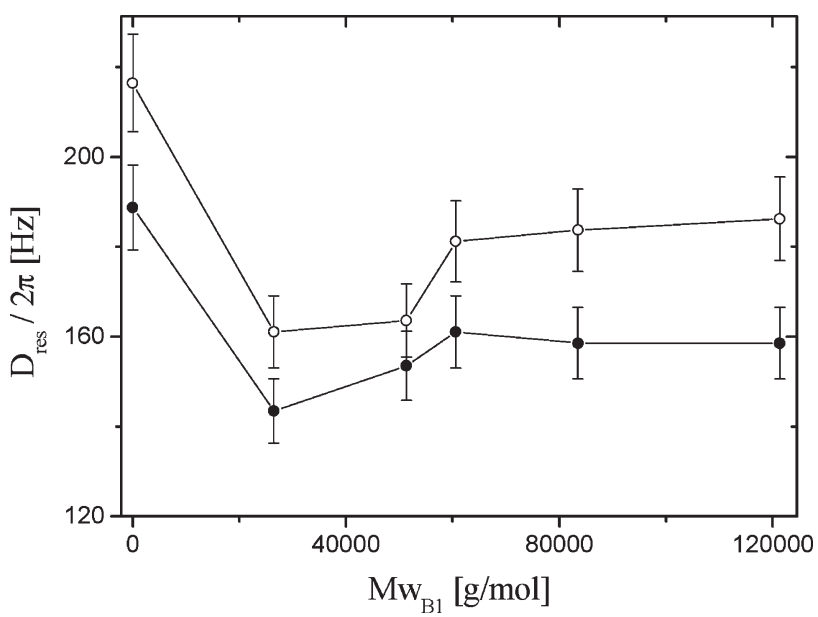

Figure 3. $D_{\text {res }}$ values as a function of molecular weight of $\mathrm{B}_{1}$, for $\mathrm{A}_{4}$ (open symbols) and $\mathrm{A}_{3}$ (filled symbols) cross-linkers. Straight lines connecting data serve as a guide to the eye.

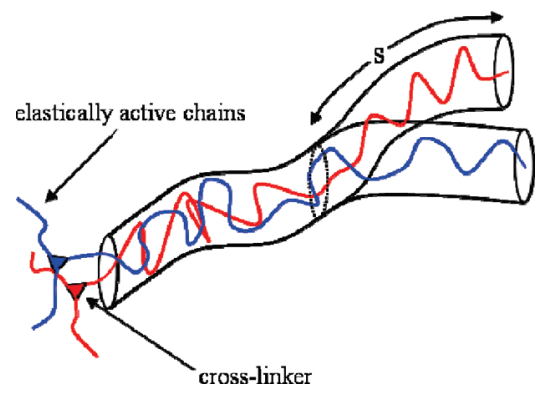

Figure 4. Pendant chain and its confining tube. A tube-like region of topological constraint arises around the pendant chains due to the topological constraints of network chains in its neighborhood. Here s is the fractional distance back along the primitive path the free end has been retracted at time $\tau(\mathrm{s})$. According to the arm retraction process, the time required to retract the free end to the branching point fully $(s=1)$ grows roughly exponentially with the length of the pendant chain. At the time-scale of the NMR experiments the pendant material near the branching point can partially relax its configuration through a Rouse-like dynamics, but can not completely "forget" the early configuration.

here we can neglect its contribution. This approximation is based on the fact that the molecular weight of the elastically active chains is similar to the molecular weight between entanglements in melts ${ }^{32}$ and that a similar fraction of trapped entanglements can be expected for all the networks of a given functionality.

(c) Transiently trapped entanglements involving the unrelaxed fraction of pendant material. As compared with other relaxational processes in the system, the dynamics of $B_{1}$ chains is very slow. Then, at the time scale of NMR experiments it can be expected that a fraction of the pendant material render a nonzero average dipolar coupling, acting in this way as the elastically active chains. To determine the fraction of transiently trapped pendant material we must take into account that the relaxation of these defects is produced via the arm retraction process. This mechanism of relaxation is illustrated in the scheme of Figure 4. According to this tube model approach for the pendant chain relaxation, at time $\tau(s)$ a fraction $s$, near the free end of the pendant is relaxed. Then, at time $\tau(s)$ the Rouse modes are unable to relax the topological anisotropy imposed by the network on the fraction $(1-s)$ of the pendant chain closer to the cross-linking point.

To determine the average fraction of tube not visited by the pendant chain end, here we have employed a modified version of 


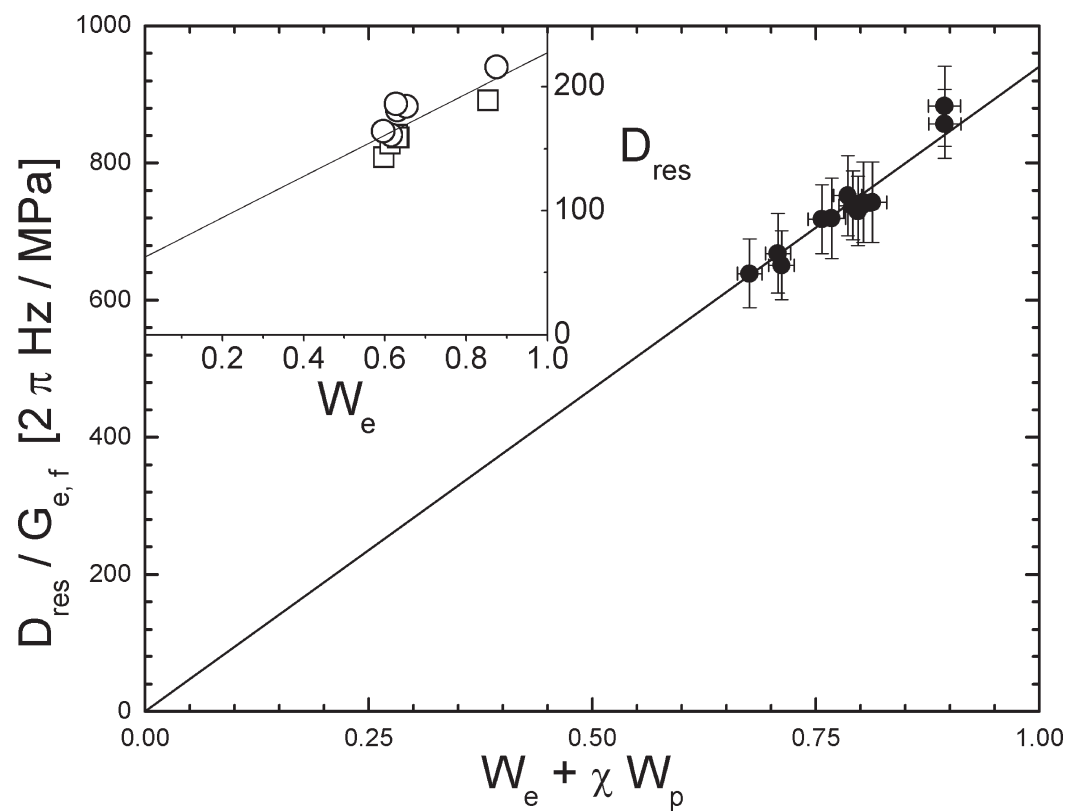

Figure 5. $D_{\text {res }}$ normalized with the storage modulus $G_{\mathrm{e}, \mathrm{f}} \mathrm{vs} W_{e}+\chi W_{p}$. The straight line indicates a linear correlation between both quantities. Inset: $D_{\text {res }}$ vs $W_{e}$ for $\mathrm{A}_{3}$ (squares) and $\mathrm{A}_{4}$ networks (circles). Note that when plotted as a function of $\mathrm{W}_{e}, D_{\text {res }}$ values do not extrapolate to zero at $W_{e}=0$.

the parameter free theory of Milner and McLeish. ${ }^{5,6}$ The main difference between our model and the Milner-McLeish model is the effect of dynamic dilution. Since here the concentration of $\mathrm{B}_{1}$ chains is relatively small (implying that only a small fraction of these chains is involved in self-entanglements), we consider the arm retraction process in a Pearson-Helfand potential ${ }^{16,33}$ rather than in a diluted Milner-McLeish potential. In Appendix II, we present the parameter free model employed here to determine the fraction of unrelaxed pendant material in the network.

In Table 1, we show the calculated values of the fraction of unrelaxed pendant material in the network, $\chi$, for the different systems studied here (see Appendix II). For networks prepared without monofunctional chains $\sim 85 \%$ of the pendant material is completely relaxed. In this case, since the dangling material is formed by partially reacted $\mathrm{B}_{2}$ chains, the molecular weight of the pendants is similar to the molecular weight between entanglements, $M_{e}$. Then, at the time-scale of the DQ-NMR experiments most of the partially reacted $\mathrm{B}_{2}$ chains (pendants) are completely relaxed ${ }^{13}$. On the other hand, for networks prepared with long pendant chains $\mathrm{B}_{1}\left(M_{\mathrm{wB} 1} \gg M_{e}\right)$, only $\sim 50 \%$ of the pendant material is relaxed. Then, in order to describe the DQ NMR response, both elastically active and unrelaxed pendants should be considered.

Taking into account both, elastic and unrelaxed pendant material, the RDC moment can be expressed as $D_{\text {res }} \sim W_{e}+$ $\chi W_{p}$. Note that small contents of pendant material $\left(W_{p} \sim 0\right)$ or pendant chains of small molecular weight $\left(n_{e} \sim 1\right)$, leads to $D_{\text {res }} \sim W_{e}$, as expected.

Figure 5 shows the experimental data of $D_{\text {res }}$ vs theoretical predictions for $W_{e}+\chi W_{p}$. In order to remove the dependence of the network elasticity on cross-linker functionality, $D_{\text {res }}$ has been normalized with the shear equilibrium modulus of the networks without monofunctional chains $\mathrm{B}_{1}, G_{e, 3}=0.214 \mathrm{MPa}$ and $G_{e, 4}=$ $0.252 \mathrm{MPa}$ for $\mathrm{A}_{3}$ and $\mathrm{A}_{4}$ cross-linkers, respectively ${ }^{24}$. Through this normalization, we take into account both, the effect of trapped entanglements and the functionality of the cross-linkers. In Figure 5, a linear correlation between $D_{\text {res }} / G_{e, f}$ and $W_{e}+\chi W_{p}$ is clearly observed, indicating the influence of the pendant material on the network elasticity. In addition, it is also observed that data collapse on a single curve irrespective of network functionality when the appropriate normalization through the shear storage modulus associated to each set of networks is employed. In the inset of Figure 5, we plot $D_{\text {res }}$ as a function of $W_{e}$ to more clearly notice the effect of the entanglements. Note that when the contribution of the unrelaxed pendant material is not taken into account the data do not extrapolate to zero for $W_{e}=0$, indicating the presence of trapped entanglements at the DQ NMR time scale.

\section{Conclusions}

Although limited in the time scale of analysis, DQ NMR experiments can be employed to determine important information about the network structure. However, the contribution of the defects must be taken into consideration. Whatever being the method of sample preparation, the presence of soluble and pendant defects is completely unavoidable. Since the dynamics of relaxation of branched soluble molecules and pendant material is quite slow, in addition to elastically active chains, the unrelaxed fraction of entanglements near the branching point also contributes to render a nonzero average dipolar coupling and should be taken into account in the data analysis.

Although at present it has been possible to prepare simple branched polymers of well-defined structure in a wide number of systems, the preparation of model networks with well-defined structure of defects has been almost entirely focused in PDMS. Then, in order to test the universality of the behavior observed here, it should be desirable to test the dynamic and DQ NMR response of polymer networks other than PDMS.

Finally, it should be emphasized that although here we have employed the Pearson-Helfand potential to determine the contribution of transiently trapped entanglements, since only a small fraction of pendant material is relaxed at the time-scale of the experiments, $\chi$ results only weakly dependent on the strength of the arm retraction potential. In this sense, as compared with the first-passage time of the pendants, the time-scale of the NMR experiments is too small to determine the strength of the arm retraction potential.

Acknowledgment. We express our gratitude to the Universidad Nacional del Sur, to the Research Council of Argentina: CONICET, ANPCyT, and to SeCyT-UNC, MCyT Córdoba, and the Partner Group for NMR Spectroscopy 
with High Spin Polarization FaMAF-Max Planck Institute for Polymer Research, Mainz, Germany which supported this work. D.A.V. also thanks the USAMI program through the National Science Foundation and Princeton University for financial support.

\section{Appendix I}

Here we calculate the weight average concentration of elastically active chains $\left(W_{e}\right)$, soluble $\left(W_{s}\right)$, and pendant material $\left(W_{p}\right)$ for $\mathrm{A}_{f}+\mathrm{B}_{2}+\mathrm{B}_{1}$ systems (for more details see ref 24).

Let $r$ be the stoichiometric imbalance, defined as $r=f\left[A_{f}\right] /$ $\left(2\left[B_{2}\right]+\left[B_{1}\right]\right)$. Then, according to the Miller-Makosco-Vallés model, ${ }^{24,34}$ the average weight fraction of elastically active $\left(W_{e}\right)$, soluble $\left(W_{s}\right)$, and pendant $\left(W_{p}\right)$ material can be calculated as follows:

$$
\begin{gathered}
W_{e}=W_{A f}\left[(1-\alpha)^{3}+\sum_{i=2}^{f-1}\left(\begin{array}{c}
f \\
i
\end{array}\right)\left(\frac{i}{f}\right) \alpha^{f-1}(1-\alpha)^{i}\right] \\
+W_{B 2}(1-\beta)^{2} \\
W_{s}=W_{A f} \alpha^{3}+W_{B 2} \beta^{2}+W_{B 1} \beta
\end{gathered}
$$

and

$$
W_{p}=1-\left(W_{s}+W_{e}\right)
$$

Here $W_{\mathrm{B} 2}, W_{\mathrm{B} 1}$, and $W_{\mathrm{A} f}$ are the initial weight fractions of $\mathrm{B}_{2}, \mathrm{~B}_{1}$, and $\mathrm{A}_{f}$ molecules in the reaction mixture, respectively, and $\alpha$ represents the probability that, looking out from an $A$ group, the reaction leads to a finite or dangling chain rather than to the infinite network and depends on the functionality of the network. In our case,

$$
\alpha=\frac{1-r v p^{2}}{r v p^{2}}
$$

for trifunctional networks and

$$
\alpha=\left(\frac{1}{r v p^{2}}-\frac{3}{4}\right)^{1 / 2}-\frac{1}{2}
$$

for tetra-functional networks.

Similarly, $\beta$ represents the probability that, looking out from a $B$ group, the reaction leads to a finite or dangling chain rather than to the infinite network:

$$
\beta=1+r p\left(\alpha^{f-1}-1\right)
$$

In these equations $v$ is the initial fraction of $\mathrm{B}$ reactive groups belonging to difunctional molecules $\mathrm{B}_{2}$

$$
v=\frac{2\left[B_{2}\right]}{2\left[B_{2}\right]+\left[B_{1}\right]}
$$

and $p$ is the extent of reaction that can be determined through the previous equations and the experimental determination of the fraction of solubles $W_{s}$. If the content of $\mathrm{B}_{1}$ chains is not too large (below $\sim 20 \mathrm{wt} \%$ ) the extent of reaction is typically between $p=0.85$ and $p=0.95$.

Note that in these systems, the amount of pendant and soluble material is a function not only of the stoichiometric imbalance but also of the proportion of $\mathrm{B}_{1}$ groups added to the system.

\section{Appendix II}

The contribution of unrelaxed pendant material to the elastic response in NMR experiments can be determined as follows. Taking into account the probability distribution of the primitive path lengths at equilibrium and the tube diameter, the free end of the pendant chain can be thought to be undergoing Brownian motion in a suitable potential field. The probability distribution for arm retraction is then the solution of the Smoluchowski equation for the probability of the deepest penetration as a function of time. The Pearson-Helfand model predicts that the potential has the following quadratic form: $: 6,13,16,33$

$$
U_{P H}=\frac{15}{8} n_{e} s^{2}
$$

Here $n_{e}$ is the number of entanglements per pendant chain, and $s$ $(0<s<1)$ is the fractional distance back along the primitive path the free end has retracted. At long times, $t>\tau\left(s=1 / n_{e}{ }^{1 / 2}\right)$, the slow relaxation process is controlled by the so denominated "First-Passage-Time" $\tau_{\mathrm{s}}(s)$. The solution to the first passage time for this problem can be expressed as follows: ${ }^{13}$

$$
\tau_{e}\left(s, n_{e}\right)=-\frac{15}{16} \frac{I \pi^{3} n_{e}^{3} \tau_{e}}{\alpha} \operatorname{erf}\left(I \sqrt{\alpha n_{e} s}\right)
$$

where $I=-1^{1 / 2}$ and $\operatorname{erf}(x)$ is the error function. ${ }^{13}$ At short times, $t<\tau\left(s=1 / n_{e}{ }^{1 / 2}\right)$, the influence of the cross-linked point can be neglected and the free end moves under the action of many Rouse modes, with no influence of the potential. In this case, the relaxation process is dominated by a one-dimensional Rouselike dynamic characterized by $\tau_{f}(s)^{6}$, where

$$
\tau_{f}(s)=\frac{225 \pi^{3}}{256} \tau_{e} n_{e}^{4} s^{4}
$$

Here $\tau_{e}$ is the Rouse time between entanglements ${ }^{35}$ and can be determined through independent experiments with linear chains. ${ }^{6}$ The relaxation time $\tau(s)$ can be determined through a crossover formula taking into account both time scales. ${ }^{5,6}$

Thus, the average fraction $\chi_{0}$ of tube not visited by the pendant chain end (unrelaxed) at time $t$ is

$$
\chi_{0} \approx \int_{0}^{1} \mathrm{~d} s \exp \left[-t / \tau\left(s, n_{e}\right)\right]
$$

Taking into account the molecular weight distribution of the pendant material, the fraction of unrelaxed material at time $t=$ $\tau_{\alpha}$ can be determined as (here $\tau_{\alpha} \sim 1 \mathrm{~ms}$ ):

$$
\chi=\int_{0}^{\infty} \mathrm{d} n_{e} \int_{0}^{1} \mathrm{~d} s P\left(n_{e}\right) \exp \left[-\tau_{\alpha} / \tau\left(s, n_{e}\right)\right]
$$

Here $P\left(n_{e}\right)$ is the relative volume fraction of defects with average number of entanglements $n_{e} . P\left(n_{e}\right)$ can be determined through the molecular weight distribution of $\mathrm{B}_{1}$ and $\mathrm{B}_{2}$ chains and the mean field description of the network structure $\left(\int_{0}^{\infty} \mathrm{d} n_{e} P\left(n_{e}\right)=1\right)$

\section{References and Notes}

(1) Watanabe, H. Prog. Polym. Sci. 1999, 24, 1253-1403.

(2) Doi, M.; Edwards, S. F. The Theory of Polymer Dynamics; Clarendon Press: Oxford, U.K., 1986.

(3) Marrucci, G. J. Polym. Sci., Polym. Phys. Ed. 1985, 23, 159-177.

(4) Ball, R. C.; McLeish, T. C. B. Macromolecules 1989, 22, 1911-1913.

(5) McLeish, T. C. B. Adv. Phys. 2002, 51, 1379-1527.

(6) Milner, S. T.; McLeish, T. C. B. Macromolecules 1997, 30, 2159-2166 
(7) Vega, D. A.; Sebastian, J. M.; Rusell, W. B.; Register, R. A. Macromolecules 2002, 35, 169-177.

(8) Kapnistos, M.; Semenov, A.; Vlassopoulos, D.; Roovers, J. J. Chem. Phys. 1999, 11, 1753-1759.

(9) McLeish, T. C. B.; Larson, R. G. J. Rheol. 1998, 42, 81-110.

(10) Frischknecht, A. L.; Milner, S. T. Macromolecules 2000, 33, 9764-9768.

(11) McLeish, T.; Allgaier, J.; Bick, D.; Bishko, G.; Biswas, P.; Blackwell, R.; lottiere, B.; Clarke, N.; Gibbs, B.; Grovers, D.; Hakiki, A.; Heenan, R.; Johnson, J.; Kant, R.; Read, D.; Young, R. Macromolecules 1999, 32, 6734-6758.

(12) Urayama, K.; Yokoyama, K.; Kohjiya, S. Macromolecules 2001, 34, 4513-4518.

(13) Vega, D. A.; Gómez, L. R.; Roth, L. E.; Ressia, J. A.; Villar, M. A.; Vallés, E. M. Phys. Rev. Lett. 2005, 95, 166002.

(14) Urayama, K.; Kawamura, T.; Kohjiya, S. Macromolecules 2001, 34, 8261-8269.

(15) Larsen, A. L.; Hansen, K.; Sommer-Larsen, P.; Hassager, A.; Bach; Ndoni, S.; Jørgensen, M. Macromolecules 2003, 36, 10063-10070.

(16) Pearson, D. S.; Helfand, E. Macromolecules 1984, 17, 888-895.

(17) Schmidt-Rohr, K.; Spiess, H. W. Multidimensional Solid-State NMR and Polymers; Academic Press: London, 1996.

(18) Cohen-Addad, J. P. J. Chem. Phys. 1973, 60, 2440-2453.

(19) Vega, D. A.; Villar, M. A.; Vallés, E. M.; Steren, C. A.; Monti, G. A. Macromolecules 2001, 34, 283-288.

(20) Graf, R.; Heuer, A.; Spiess, H. W. Phys. Rev. Lett. 1998, 80, 5738-5741.
(21) Voda, M. A.; Demco, D. E.; Perlo, J.; Orza, R. A.; Blumich, B. J. Magn. Reson. 2005, 172, 98-109.

(22) Saalwachter, K. Prog. NMR Spectrosc. 2007, 51, 1-35 and references contained therein.

(23) Acosta, R. H.; Vega, D. A.; Villar, M. A.; Monti, G. A.; Vallés, E. M. Macromolecules 2006, 39, 4793-4801.

(24) Villar, M. A.; Bibbo, M. A.; Vallés, E. M. Macromolecules 1996, 29, 4072-4080.

(25) Villar, M. A.; Vallés, E. M. Macromolecules 1996, 29, 4081-4089.

(26) Urayama, K.; Miki, T.; Takigawa, T.; Kohjiya, S. Chem. Mater. 2004, 16, 173-178.

(27) Ernst, R. R.; Bodenhausen, G.; Wokaun, A., Principles of Nuclear Magnetic Resonance in One and Two Dimensions; Clarendon Press: Oxford. U.K., 1987.

(28) Baum, J.; Pines, A. J. Am. Chem. Soc. 1986, 108, 7447-7454.

(29) Fisher, E.; Grinberg, F.; Kimmich, R.; Hafner, S. J. Chem. Phys. 1998, 109, 846-854.

(30) Saalwachter, K.; Ziegler, P.; Spyckerelle, O.; Haidar, B.; Vidal, A.; Sommer, J. J. Chem. Phys. 2003, 119, 3468-3482.

(31) Saalwachter, K.; Herrero, B.; López-Manchado, M. A. Macromolecules 2005, 38, 9650-9660.

(32) Fetters, L. J.; Lohse, D. J.; Richter, D.; Witten, T. A.; Zirkel, A. Macromolecules 1994, 27, 4639-4647.

(33) Shanbhag, S.; Larson, R. G. Phys. Rev. Lett. 2005, 94, 076001

(34) Miller, D. R.; Vallés, E. M.; y Macosko, C. W. Polym. Eng. Sci. 1979, 19, 272-283.

(35) For PDMS at $T=273 \mathrm{~K}$, we have $\tau_{\mathrm{e}} \sim 1.2 \times 10^{-6}$ s (see also ref 13 ). 